\title{
Planar Sectoral Antenna for IR-UWB Localization with Minimal Range Estimation Biasing
}

\author{
Quinten Van den Brande, Student Member, IEEE, Sam Lemey, Member, IEEE, \\ and Hendrik Rogier, Senior Member, IEEE
}

\begin{abstract}
A planar sectoral antenna is presented, optimized for highly precise Impulse-Radio Ultra-Wideband (IR-UWB) indoor localization with minimal range estimation biasing. By judiciously combining two planar inverted-F antenna (PIFA) elements into one footprint, a large half-power beamwidth (HPBW) is obtained in the [3.2448 - 4.7424] GHz band, thereby covering channels 1 to 4 of the IEEE 802.15.4a-2011 standard. Through system-level optimization, the system fidelity factor (SFF) is maximized for a minimal value of at least $90 \%$ within its entire HPBW, while minimizing the orientation-specific range estimation biasing down to $10 \mathrm{~mm}$. To validate the antenna performance, measurements have been performed in both the frequency and time domain, showing a HPBW larger than $120^{\circ}$ in the complete frequency-band of operation and an SFF larger than $90 \%$ and range biasing lower than $4 \mathrm{~mm}$ within the antenna's HPBW.
\end{abstract}

Index Terms-IR-UWB, Air-Filled Substrate-IntegratedWaveguide, High Efficiency, Sectoral Antenna, Range Biasing

\section{INTRODUCTION}

W ITH the rapid advent of Industry 4.0 in high-tech industrial environments, tangible research results on state-of-the-art sensor systems are of major importance to facilitate a steady transition into this new industrial revolution. A great example can be found in the domain of indoor localization, demonstrating major advantages in different sectors and on different scales [1], [2]. Here, Impulse-Radio Ultra-Wideband (IR-UWB) localization systems yield superior accuracy, attributed to their resilience against narrowband interference and detrimental multipath effects [3]. Furthermore, because of the development of low-cost and accurate IR-UWB localization sensor platforms [4], IR-UWB indoor localization has become a driving force for innovation in many applications in healthcare [5], robot navigation [6], [7], etc. However, with IR-UWB localization systems entering the stage of mass production, new research challenges associated with the integration of IR-UWB antennas in practical indoor localization systems emerge to ensure cm-level-precision accuracy to a large number of users in the harsh and challenging Industry 4.0 environment [8], [9].

The use of sub-nanosecond pulses in IR-UWB localization systems imposes a substantially different antenna design procedure when compared to narrowband technology. As stated in [10], [11], both the frequency-domain and system-level performance of the antenna should be optimized, where the latter focuses primarily on the pulse distortion introduced by the antenna system. However, recent studies have demonstrated that not only pulse distortion, but also the orientation- dependent time delay introduced by the antenna should be investigated at a system level [12]-[15]. These delays were investigated in [12], [13], [15] through time-domain measurements, showing an antenna-induced orientation-dependent range estimation bias up to $30 \mathrm{~cm}$. In [14], the influence of this range estimation bias was studied for angle-of-arrival estimation. Furthermore, [16]-[19] report that certain IRUWB hardware platforms exhibit a correlation between range estimation bias and received power, thereby requiring minimal antenna gain variation within the targeted field of view.

In this work, a planar sectoral coupled Planar Inverted-F Antenna (PIFA) for IR-UWB localization is proposed, operational in the [3.2448 - 4.7424] GHz band, thereby covering channels 1 through 4 of the IEEE 802.15.4a-2011 standard [20]. Inspired by the topology in [21] and by means of a rigorous analysis and optimization process, both in the frequencydomain and on a system level, a large HPBW and minimal range estimation biasing within that HPBW is obtained. The antenna performance is validated by both frequency- and timedomain measurements, thereby confirming the potential of the proposed antenna for IR-UWB indoor localization systems.

A plethora of antennas for IR-UWB localization have been extensively analyzed in literature, both with omnidirectional [22]-[24] and hemispherical [25]-[28] radiation properties. Analyzing the frequency domain radiation characteristics of [22]-[24], it is apparent that an omnidirectional antenna is not suited as a sector antenna. To mitigate interference in sectorized network topologies, several omnidirectional antennas were equipped with a reflector to shape the radiation pattern towards the targeted cell area [25], [26]. However, this approach creates highly directive antennas and may cause strong variations in the radiation pattern as a function of frequency. In [27], a hemispherical antenna is presented, achieving a HPBW larger than $90^{\circ}$ and showing a fidelity factor of $80 \%$ in broadside. Unfortunately, a gain as low as $-15 \mathrm{dBi}$ is reported, rendering it unsuitable for the envisioned application scenario. In terms of time-domain characteristics, most research imposes a design requirement of a system fidelity factor (SFF) higher than $50 \%$, as defined in [10]. However, the state-of-the-art IR-UWB antennas easily reach a measured SFF higher than $80 \%$ [22]-[24], [27]. In [26], [28], SFFs up to $91 \%$ and $95 \%$ are reported, respectively. Furthermore, a ranging estimation bias of $50 \mathrm{~mm}$ is reported in [28], showing that a high system fidelity factor does not necessarily result in a low ranging estimation bias and that specific system-level timedomain optimization is required to optimize this parameter. Alternatively, in terms of narrowband topologies, magnetic 
dipoles, as analyzed in [21], provide highly beneficial radiation properties for sectorized antenna arrays. Despite the favorable radiation characteristics, the impedance bandwidth of $2 \%$ is not sufficient for IR-UWB applications. However, by adopting an air-substrate PIFA topology and leveraging mode-bifurcation, ultra-wideband operation is achieved. Furthermore, a time-domain optimization is performed utilizing an in-house modular full-wave/circuit co-optimization framework [29] to maximize the system fidelity factor and minimize the range estimation bias.

The remainder of this letter is organised as follows. In Section II, both the frequency- and time-domain antenna design requirements are discussed. Section III elaborates on the antenna topology, its operation principle and summarizes the final antenna specifications. Next, Section IV provides an overview of both the frequency- and time-domain simulation and measurement results. Finally, a conclusion is presented in Section V.

\section{DESIGN REQUiREMENTS}

A sectoral IR-UWB anchor antenna is designed for IRUWB localization applications in the $[3.2448-4.7424] \mathrm{GHz}$ frequency band. Thereby, the antenna covers channels 1 to 4 of the IEEE 802.15.4a-2011 standard, frequently adopted in academic and commercial localization applications [30][33]. In the following, all design requirements in the frequency domain are imposed over the entire specified frequency band. The magnitude of the reflection coefficient, $\left|S_{1,1}\right|$, with respect to $50 \Omega$, should remain below $-10 \mathrm{~dB}$. Furthermore, a total antenna efficiency above $85 \%$ is targeted to maximize range and minimize power consumption. Finally, in order to comfortably provide a $360^{\circ}$ coverage in a three-element sectorized antenna array, while minimizing inter-antenna interference, a HPBW of $120^{\circ}$ in the azimuth plane and a front-to-back-ratio (FTBR) larger than $10 \mathrm{~dB}$ is imposed on the unit antenna element.

As mentioned in [10], [11], time-domain optimization is imperative to achieve robust and excellent system performance in IR-UWB localization applications. Special care is devoted to minimizing pulse distortion and orientation-specific range estimation biasing errors, introduced by the antenna system. The pulse distortion is characterized the system fidelity factor (SFF), defined by [10]

$$
S F F=\max _{t}\left|\frac{\int_{t_{0}}^{t_{n}} s(\tau) x(\tau+t) d \tau}{\sqrt{\int_{t_{0}}^{t_{n}} s^{2}(\tau) d \tau \int_{t_{0}}^{t_{n}} x^{2}(\tau) d \tau}}\right|,
$$

with $s(t)$ and $x(t)$ the input and output pulse, respectively. The algorithm used to estimate the range and, hence, the orientation-specific biasing error, $d_{e s t}(\theta, \phi)$, is defined by

$$
d_{\text {est }}(\theta, \phi)=\left(t_{\max }(\theta, \phi)-t_{\max }(0,0)\right) c,
$$

where $t_{\max }$ is the time delay that maximizes the SFF for a certain orientation $(\theta, \phi)$ and $c$ is the speed of light. As such, for a given antenna system, the output pulse needs to be calculated for each orientation of the antenna under test (AUT). To guarantee high-precision localization with minimal orientation-specific biasing errors, both an SFF higher than

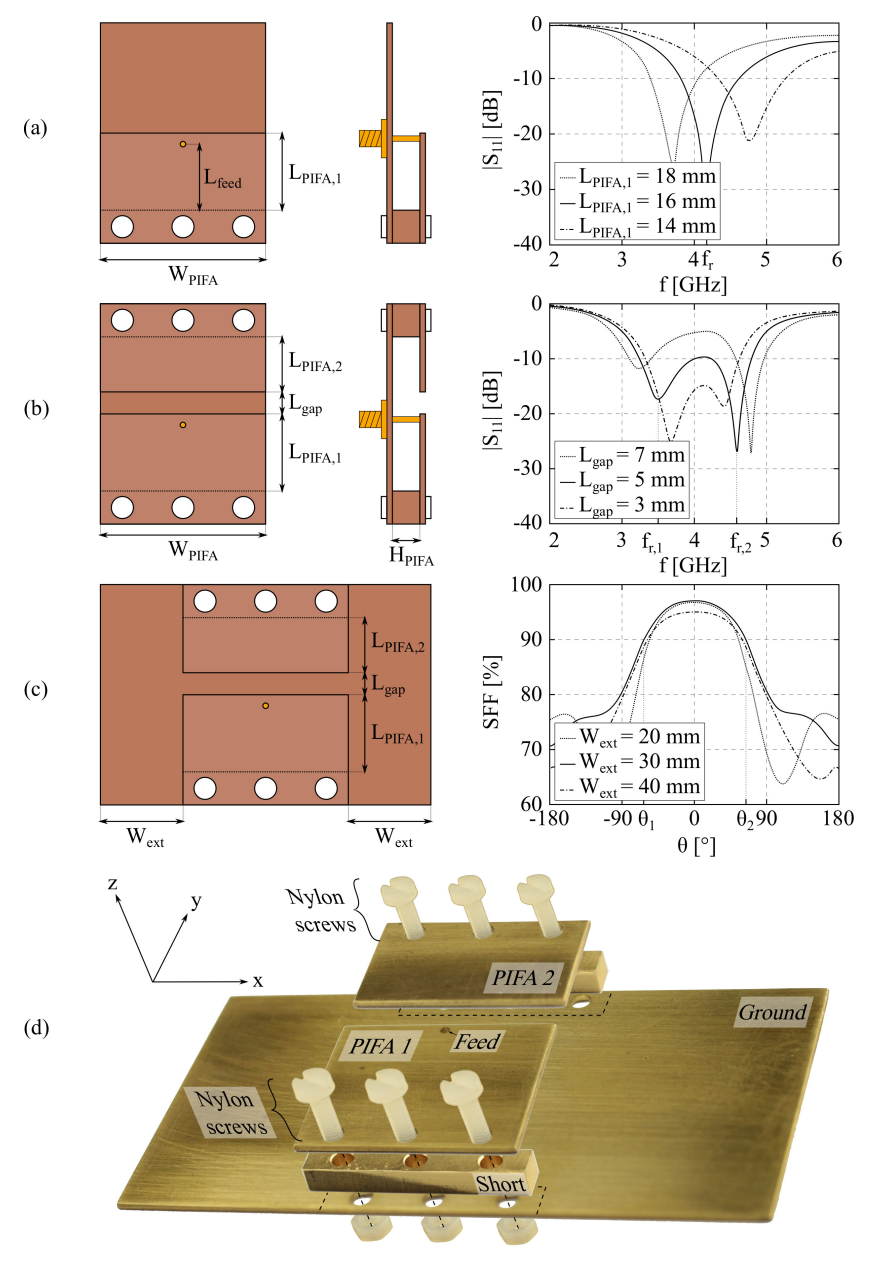

Figure 1. Design process of the coupled PIFA IR-UWB antenna: Single PIFA (a), coupled PIFA (b), ground plane extension (c) and exploded view of the prototype (d). The parameter sweeps are obtained with the final antenna dimensions, specified in Table I, unless stated otherwise.

$90 \%$ and a distance estimation biasing error, $\left|d_{e s t}\right|$, lower than $10 \mathrm{~mm}$ are imposed.

\section{Sectoral Coupled Planar Inverted-F ANTENNA: TOPOLOGY AND OPERATION PRINCIPLE}

\section{A. Ultra-Wideband Half-Power Beamwidth Optimization}

A suitable antenna topology with favorable inherent radiation properties is required and needs to be tailored to satisfy the aforementioned design requirements. A promising candidate is the microstrip patch antenna, because of its low profile, wide HPBW and large FTBR [34]-[38]. In addition, [39] demonstrates that the HPBW can be further enhanced by cutting the patch in half along its E-plane and shorting the patch along this cut. Although the mode profile inside the resulting planar inverted-F antenna is identical to half of the TM01-mode in the original patch, the radiation characteristics have slightly changed. By only retaining half of the TM01mode, the contribution of both sidewalls to the radiation pattern increases substantially and, as a result, the HPBW enlarges compared to a patch antenna [39]. 
Evidently, the PIFA exhibits advantageous radiation properties and, as such, is selected as the starting point for this design. However, despite the potential of the PIFA, its inherent narrowband behaviour remains a major drawback for IRUWB applications. To resolve this issue, a technique called mode-bifurcation [11] is exploited. The resulting coupled PIFA antenna topology, its design flow and implementation is illustrated in Fig. 1, depicting both the top and side views in each step, as well as the effect of antenna dimensions on the relevant antenna characteristics.

Initially, as depicted in Fig. 1(a), a single PIFA element, fed by a coaxial feed, is impedance-matched to $50 \Omega$ at a frequency $\mathrm{f}_{\mathrm{r}}=4 \mathrm{GHz}$. By optimizing the width $\left(\mathrm{W}_{\mathrm{PIFA}}\right)$, length $\left(\mathrm{L}_{\mathrm{PIFA}, 1}\right)$ and feed position $\left(\mathrm{L}_{\text {feed }}=10.5 \mathrm{~mm}\right)$ of the PIFA, the antenna exhibits a resonance at $4 \mathrm{GHz}$. Next, a second identical PIFA element is added to the antenna footprint, as shown in Fig. 1(b). The addition of the second PIFA element induces mode-bifurcation, resulting in two distinct resonant peaks, $\mathrm{f}_{\mathrm{r}, 1}$ and $\mathrm{f}_{\mathrm{r}, 2}$, with $\mathrm{f}_{\mathrm{r}, 1}<\mathrm{f}_{\mathrm{r}}<\mathrm{f}_{\mathrm{r}, 2}$. The frequencies $f_{r, 1}$ and $f_{r, 2}$ and, hence, the impedance bandwidth are controlled by varying the amount of coupling between both PIFA elements. By carefully choosing the PIFA height $\left(\mathrm{H}_{\mathrm{PIFA}}\right)$, the gap between both PIFA elements ( $\mathrm{L}_{\text {gap }}$ ) and their respective resonant frequencies, controlled by $\mathrm{L}_{\mathrm{PIFA}, 1}$ and $\mathrm{L}_{\mathrm{PIFA}, 2}$, the shift in resonant frequencies can be adjusted to realize a reflection coefficient, $\left|S_{1,1}\right|$, below $-10 \mathrm{~dB}$ in the complete frequency band of operation. Note that the second PIFA element is not fed directly and is only excited by proximity coupling with the first PIFA element. Additionally, remark that varying $L_{g a p}$ changes the total antenna length since we keep the values of $L_{P I F A, 1}$ and $L_{P I F A, 2}$ fixed during the process. Finally, as illustrated by Fig. 1(c), a ground plane extension is added to tailor the radiation pattern to the intended deployment scenario. Tuning $\mathrm{W}_{\text {ext }}$ allows to optimize for a HPBW in the azimuth plane (xz-plane) of $120^{\circ}$, while maintaining a FTBR larger than $10 \mathrm{~dB}$.

\section{B. Coupled Planar Inverted-F Antenna and Time-Domain Performance}

In addition to the conventional frequency-domain optimization, a system-oriented time-domain optimization is required to guarantee robust and accurate performance for IR-UWB localization applications. This optimization is performed based on a pulse defined by [20]

$$
s(t)=\cos \left(2 \pi f_{0} t\right) \frac{2}{\pi \sqrt{T_{p}}} \frac{\cos \left(1.5 \pi t / T_{p}\right)+\frac{\sin \left(0.5 \pi t / T_{p}\right)}{2 t / T_{p}}}{1-\left(2 t / T_{p}\right)^{2}},
$$

with $f_{0}=4.25 \mathrm{GHz}$ the pulse carrier frequency and $T_{p}=0.92$ ns the pulse duration, thereby covering channels 1 through 4 . The pulse is transmitted by a coupled half-mode cavity backed slot antenna, developed in [40], and is received by the antenna under test (AUT) presented in this work. Finally, an in-house modular full-wave/circuit co-optimization framework [29] is utilized to obtain the required time-domain simulation results.

On the one hand, an extensive parameter analysis revealed that the SFF and, hence, the pulse distortion, is predominantly affected by the mode-bifurcation. On the other hand, the
Table I

FINAL ANTENNA DIMENSIONS (AND FABRICATION TOLERANCES).

\begin{tabular}{|c|c|c|c|}
\hline Dimension & Value $[\mathbf{m m}]$ & Dimension & Value $[\mathbf{m m}]$ \\
\hline \hline $\mathrm{W}_{\mathrm{PIFA}}$ & $30.0( \pm 0.5)$ & $\mathrm{L}_{\text {feed }}$ & $18.7( \pm 0.1)$ \\
\hline $\mathrm{L}_{\mathrm{PIFA}, 1}$ & $20.1( \pm 0.5)$ & $\mathrm{L}_{\text {gap }}$ & $5.0( \pm 0.5)$ \\
\hline $\mathrm{L}_{\mathrm{PIFA}, 2}$ & $12.4( \pm 0.5)$ & $\mathrm{W}_{\text {ext }}$ & $30.0( \pm 0.5)$ \\
\hline $\mathrm{H}_{\mathrm{PIFA}}$ & $3.55( \pm 0.1)$ & $\mathrm{t}_{\text {metal }}$ & 1.0 \\
\hline
\end{tabular}

$\mathrm{AUT}^{\prime}$ s orientation-dependent pulse distortion and the range estimation biasing error are primarily influenced by the ground plane extension. Therefore, two subsequent multi-objective optimization procedures, as defined in [11], are performed. First, a set of Pareto optimal solutions is found that minimize the pulse distortion by maximizing the SFF, while preserving the required frequency-domain properties. To this end, the resonance frequencies of the PIFA elements and their coupling are optimized by adjusting LPIFA,1, LPIFA,2 and $\mathrm{L}_{\text {gap }}$. Next, by sizing the ground plane extension $\left(\mathrm{W}_{\mathrm{ext}}\right)$, a second set of Pareto optimal solutions is obtained that maximize the orientationspecific SFF while minimizing the range estimation biasing error within the antenna's HPBW in the azimuth plane.

\section{Fabrication and Final Prototype}

Finally, the antenna performance is demonstrated by manufacturing a prototype out of brass sheets, with a thickness of $1.0 \mathrm{~mm}$, using a combination of $\mathrm{CNC}$ milling and shearing, exhibiting fabrication tolerances of $\pm 0.1 \mathrm{~mm}$ and $\pm 0.5 \mathrm{~mm}$, respectively. Nevertheless, other non-ferrous metals, such as copper or aluminum, could also be applied for the antenna production. Furthermore, diverse metalworking techniques, such as, laser cutting or metal bending, can be adopted without deteriorating the antenna performance. To assemble the different antenna parts without corrupting the antenna performance, standard M3 nylon screws and bolts are used.

The final antenna dimensions are optimized using the CST Microwave Solver. They are summarized in Table I, detailing the dimensions after optimization and the expected tolerances. An exploded view of the final antenna prototype is depicted in Fig. 1(d).

\section{Simulation and Measurement Results}

\section{A. Frequency-domain results}

The frequency-domain performance of the antenna is measured in an anechoic chamber, using a Keysight N5242a PNA-X network analyzer and a NSI-MI spherical near-field scanner. Fig. 2, depicting the reflection coefficient $\left|S_{11}\right|$ with respect to $Z_{0}=50 \Omega$, shows a good agreement between the measurement results of two prototypes and simulations. An $-10 \mathrm{~dB}$ impedance bandwidth of $1.5 \mathrm{GHz}$ is obtained around $4 \mathrm{GHz}$, yielding a fractional bandwidth of $37.5 \%$. Furthermore, results of an extensive parameter sweep have been added to Fig. 2, indicated by the gray area, to demonstrate robustness against fabrication tolerances and material variations. Fig. 3 shows a good agreement between the simulated and measured 


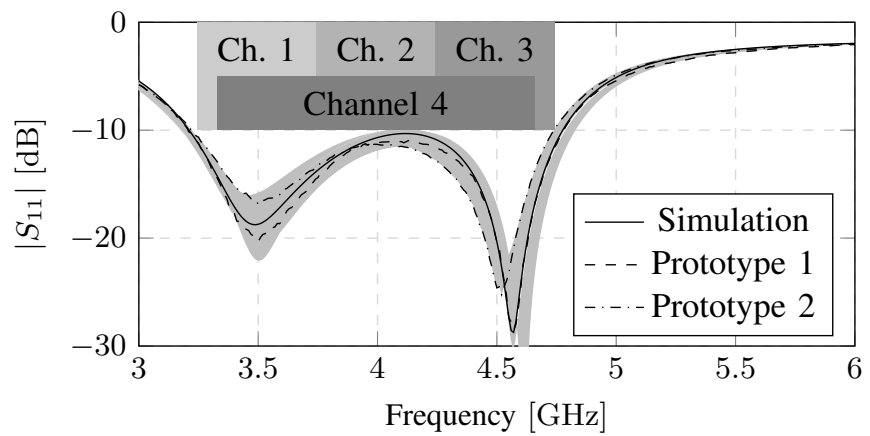

Figure 2. Simulated and measured reflection coefficient $\left|S_{11}\right|$ w.r.t. $50 \Omega$ of the final antenna (black lines) and parameter sweeps (grey area) to assess fabrication tolerances.
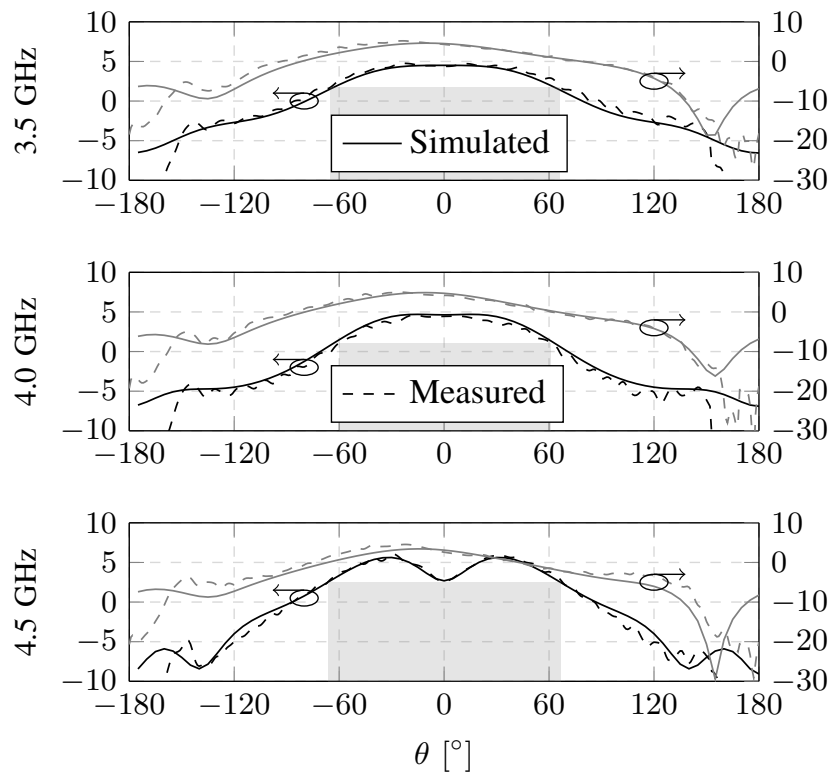

Figure 3. Simulated (solid) and measured (dashed) antenna gain [dBi] in the azimuth plane (black) and elevation plane (gray) at 3.5, 4.0 and $4.5 \mathrm{GHz}$.

gain pattern in the azimuth (xz-plane) and elevation plane (yzplane) at $3.5 \mathrm{GHz}, 4.0 \mathrm{GHz}$ and $4.5 \mathrm{GHz}$, reporting a boresight gain of $4.73 \mathrm{dBi}, 4.66 \mathrm{dBi}$ and $6.04 \mathrm{dBi}$, respectively. An HPBW larger than $120^{\circ}$ in the azimuth plane, indicated by the gray boxes, can be observed at each frequency, while maintaining an FTBR higher than $10 \mathrm{~dB}$ and a mean crosspolarization discrimination within that HPBW of $44.8 \mathrm{~dB}$, $38.6 \mathrm{~dB}$ and $39.0 \mathrm{~dB}$ at $3.5 \mathrm{GHz}, 4.0 \mathrm{GHz}$ and $4.5 \mathrm{GHz}$, respectively. Furthermore, note that special care is required when integrating this antenna into a three-element sectorized antenna array to preserve its radiation properties. Finally, a total antenna efficiency of $98.8 \%, 85.1 \%$ and $99.1 \%$ is measured at $3.5 \mathrm{GHz}, 4.0 \mathrm{GHz}$ and $4.5 \mathrm{GHz}$, respectively.

\section{B. Time-domain results}

To characterize the time-domain system performance, the measurement setup depicted in Fig. 4 is used. The antenna system consists of a fixed antenna, fully characterized in [11], and the current antenna which acts as the rotating AUT.

To compensate for any undesired pulse distortion introduced by the measurement setup, all measurements are calibrated

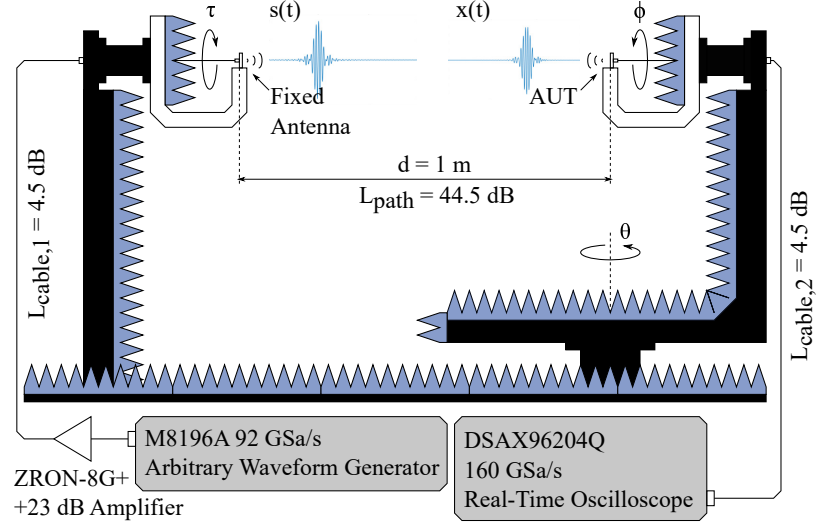

Figure 4. Measurement setup for the time-domain measurements in free-space conditions.

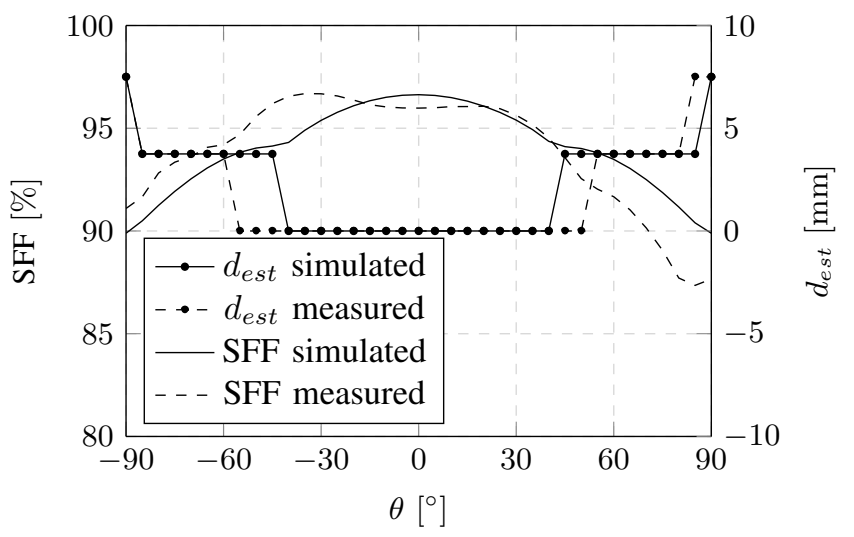

Figure 5. Simulated and measured system fidelity factor [\%] (left) and orientation-specific ranging bias [mm] (right) in the azimuth plane.

by characterizing the time-domain performance of the measurement setup (by implementing a wired through-connection without antennas) and pre-distorting the generated input pulse accordingly. The resulting time-domain results are presented in Fig. 5. A good agreement between the simulated and measured SFF can be observed, showing an SFF over $90 \%$ within the complete HPBW of the azimuth plane. Furthermore, both simulations and measurements of the ranging bias agree very well, proving a ranging bias below $4 \mathrm{~mm}$ within the complete HPBW of the azimuth plane. Moreover, an SFF above $95 \%$ and ranging bias below $8 \mathrm{~mm}$ are measured in the elevation plane for $\theta=\left[-90^{\circ} ; 90^{\circ}\right]$.

\section{CONClusion}

In this letter, a planar sectoral antenna is presented, optimized for highly precise IR-UWB indoor localization with minimal range estimation biasing. The antenna exhibits a reflection coefficient below $-10 \mathrm{~dB}$ for the frequency range [3.2448 - 4.7424] GHz. A HPBW above $120^{\circ}$ is reported for all frequencies within the frequency band of operation. Finally, an SFF higher than $90 \%$ and a ranging bias lower than $4 \mathrm{~mm}$ are measured within the HPBW of the azimuth plane. As a result, the proposed antenna is especially suited as an anchor antenna for highly accurate IR-UWB localization systems. 


\section{REFERENCES}

[1] R. Zekavat and R. M. Buehrer, Wireless Localization Using UltraWideband Signals, pp. 269-301. 2019.

[2] F. Zafari, A. Gkelias, and K. K. Leung, "A Survey of Indoor Localization Systems and Technologies," IEEE Communications Surveys Tutorials, vol. 21, no. 3, pp. 2568-2599, 2019.

[3] A. Yassin, Y. Nasser, M. Awad, A. Al-Dubai, R. Liu, C. Yuen, R. Raulefs, and E. Aboutanios, "Recent Advances in Indoor Localization: A Survey on Theoretical Approaches and Applications," IEEE Communications Surveys Tutorials, vol. 19, no. 2, pp. 1327-1346, 2017.

[4] A. R. Jimnez Ruiz and F. Seco Granja, "Comparing Ubisense, BeSpoon, and DecaWave UWB Location Systems: Indoor Performance Analysis," IEEE Transactions on Instrumentation and Measurement, vol. 66, no. 8, pp. 2106-2117, 2017.

[5] R. Bharadwaj, S. Swaisaenyakorn, C. G. Parini, J. C. Batchelor, and A. Alomainy, "Impulse Radio Ultra-Wideband Communications for Localization and Tracking of Human Body and Limbs Movement for Healthcare Applications," IEEE Transactions on Antennas and Propagation, vol. 65, no. 12, pp. 7298-7309, 2017.

[6] D. Shi, H. Mi, E. G. Collins, and J. Wu, "An Indoor Low-Cost and High-Accuracy Localization Approach for AGVs," IEEE Access, vol. 8, pp. 50085-50090, 2020.

[7] Y. Xu et al., "Robust and accurate UWB-based indoor robot localisation using integrated EKF/EFIR filtering," IET Radar, Sonar Navigation, vol. 12, no. 7, pp. 750-756, 2018.

[8] A. D. Preter, G. Goysens, J. Anthonis, J. Swevers, and G. Pipeleers, "Range Bias Modeling and Autocalibration of an UWB Positioning System," in 2019 International Conference on Indoor Positioning and Indoor Navigation (IPIN), pp. 1-8, 2019.

[9] B. Silva and G. P. Hancke, "IR-UWB-Based Non-Line-of-Sight Identification in Harsh Environments: Principles and Challenges," IEEE Trans. on Industrial Informatics, vol. 12, no. 3, pp. 1188-1195, 2016.

[10] G. Quintero, J. . Zurcher, and A. K. Skrivervik, "System Fidelity Factor: A New Method for Comparing UWB Antennas," IEEE Transactions on Antennas and Propagation, vol. 59, no. 7, pp. 2502-2512, 2011.

[11] Q. Van den Brande, S. Lemey, J. Vanfleteren, and H. Rogier, "Highly Efficient Impulse-Radio Ultra-Wideband Cavity-Backed Slot Antenna in Stacked Air-Filled Substrate Integrated Waveguide Technology," IEEE Transactions on Antennas and Propagation, vol. 66, no. 5, pp. 21992209,2018

[12] L. Zwirello, T. Schipper, M. Harter, and T. Zwick, "UWB Localization System for Indoor Applications: Concept, Realization and Analysis," Journal of Electrical and Computer Engineering, May 2012.

[13] W. Sörgel and W. Wiesbeck, "Influence of the Antennas on the UltraWideband Transmission," EURASIP Journal on Advances in Signal Processing, vol. 2005, Mar. 2005.

[14] A. Ledergerber and R. Dandrea, "Calibrating Away Inaccuracies in Ultra Wideband Range Measurements: A Maximum Likelihood Approach," IEEE Access, vol. 6, pp. 78719-78730, 2018.

[15] R. Ye, S. Redfield, and H. Liu, "High-precision indoor UWB localization: Technical challenges and method," in 2010 IEEE International Conference on Ultra-Wideband, vol. 2, pp. 1-4, 2010.

[16] M. Pelka, M. Cimdins, and H. Hellbrück, "Impact of the antenna orientation for distance estimation," Proceedings of the 3rd KuVS/GI Expert Talk on Localization, pp. 17-19, 2018.

[17] A. Ledergerber and R. D'Andrea, "Ultra-wideband range measurement model with Gaussian processes," in 2017 IEEE Conference on Control Technology and Applications (CCTA), pp. 1929-1934, 2017.

[18] V. Barral et al., "Assessment of uwb ranging bias in multipath environments," in Proceedings of the International Conference on Indoor Positioning and Indoor Navigation (IPIN), Alcala de Henares, Spain, pp. 4-7, 2016.

[19] M. Pelka, M. Cimdins, and H. Hellbrück, "Impact of the antenna orientation for distance estimation," Proceedings of the 3rd KuVS/GI Expert Talk on Localization, 2018.

[20] "IEEE Standard for Local and metropolitan area networks-Part 15.4: Low-Rate Wireless Personal Area Networks (LR-WPANs)," IEEE Std 802.15.4-2011, pp. 1-314, Sept 2011.

[21] C. Liu, S. Xiao, H. Tu, and Z. Ding, "Wide-Angle Scanning Low Profile Phased Array Antenna Based on a Novel Magnetic Dipole," IEEE Transactions on Antennas and Propagation, vol. 65, no. 3, pp. 11511162,2017

[22] B. Mohamadzade, R. B. V. B. Simorangkir, R. M. Hashmi, and A. Lalbakhsh, "A Conformal Ultrawideband Antenna With Monopole-Like Radiation Patterns), year $=2020$, volume $=68$, number $=8$, pages $=6383$ 6388,", IEEE Transactions on Antennas and Propagation.
[23] S. R. Emadian, J. Ahmadi-Shokouh, C. Ghobadi, and J. Nourinia, "Study on frequency and impulse response of novel triple band notched uwb antenna in indoor environments," AEU - International Journal of Electronics and Communications, vol. 96, pp. 93 - 106, 2018.

[24] S. Ullah, C. Ruan, M. S. Sadiq, T. U. Haq, and W. He, "High Efficient and Ultra Wide Band Monopole Antenna for Microwave Imaging and Communication Applications," Sensors, vol. 20, no. 1, 2020.

[25] A. Edalati, W. Shao, T. McCollough, and W. McCollough, "A Novel Cavity Backed Monopole Antenna with UWB Unidirectional Radiation," Progress In Electromagnetics Research, vol. 72, pp. 1-13, 2017.

[26] M. Li and K. Luk, "A differential-fed uwb antenna element with unidirectional radiation," IEEE Transactions on Antennas and Propagation, vol. 64, no. 8, pp. 3651-3656, 2016.

[27] Y. Li, X. Yang, Z. Li, L. Wang, and H. Yang, "A unidirectional cylindrical conformal monopole antenna designed for impulse radar system," IEEE Antennas and Wireless Propagation Letters, vol. 10, pp. 1397-1400, 2011

[28] D. Van Baelen, Q. Van den Brande, S. Lemey, J. Verhaevert, and H. Rogier, "Foldable all-textile cavity-backed slot antennas for personal uwb localization," Radio Science, vol. 55, no. 3, p. e2019RS006990, 2020. e2019RS006990 10.1029/2019RS006990.

[29] T. Cuyckens and H. Rogier, "Framework for the simulation of wireless power transmission over ultra wide band links in multipath propagation environments in the vicinity of the human body," International Journal of Numerical Modelling: Electronic Networks, Devices and Fields, vol. 29, no. 3, pp. 364-379, 2016.

[30] Yanchao Zhang, Wei Liu, Yuguang Fang, and Dapeng Wu, "Secure localization and authentication in ultra-wideband sensor networks," IEEE Journal on Selected Areas in Communications, vol. 24, no. 4, pp. 829-835, 2006.

[31] S. Krishnan, P. Sharma, Z. Guoping, and O. H. Woon, "A UWB based Localization System for Indoor Robot Navigation," in 2007 IEEE International Conference on Ultra-Wideband, pp. 77-82, 2007.

[32] S. J. Ingram, D. Harmer, and M. Quinlan, "UltraWideBand indoor positioning systems and their use in emergencies," in PLANS 2004. Position Location and Navigation Symposium (IEEE Cat. No.04CH37556), pp. 706-715, 2004.

[33] V. Barral, P. Surez-Casal, C. Escudero, and J. Garca-Naya, "MultiSensor Accurate Forklift Location and Tracking Simulation in Industrial Indoor Environments," Electronics, vol. 8, p. 1152, 102019.

[34] X. Chen, L. Yang, J. Zhao, and G. Fu, "High-Efficiency Compact Circularly Polarized Microstrip Antenna With Wide Beamwidth for Airborne Communication," IEEE Antennas and Wireless Propagation Letters, vol. 15, pp. 1518-1521, 2016.

[35] M. K. Ray, K. Mandal, and N. Nasimuddin, "Low-Profile Circularly Polarized Patch Antenna With Wide $3 \mathrm{~dB}$ Beamwidth," IEEE Antennas and Wireless Propagation Letters, vol. 18, no. 12, pp. 2473-2477, 2019.

[36] M. Wang, X. Zhu, Y. Guo, and W. Wu, "Compact Circularly Polarized Patch Antenna With Wide Axial-Ratio Beamwidth," IEEE Antennas and Wireless Propagation Letters, vol. 17, no. 4, pp. 714-718, 2018.

[37] K. Ng, C. H. Chan, and K. Luk, "Low-Cost Vertical Patch Antenna With Wide Axial-Ratio Beamwidth for Handheld Satellite Communications Terminals," IEEE Transactions on Antennas and Propagation, vol. 63, no. 4, pp. 1417-1424, 2015.

[38] T. P. Wong and K. M. Luk, "A wideband L-probe patch antenna array with wide beamwidth," IEEE Transactions on Antennas and Propagation, vol. 51, no. 10, pp. 3012-3014, 2003.

[39] V. Antonchik, Theory and experiment of planar inverted F-antennas for wireless communications applications. $\mathrm{PhD}$ thesis, Simon Fraser University, 2007.

[40] Q. Van den Brande, S. Lemey, H. Rogier, and J. Vanfleteren, "Coupled Half-Mode Cavity-Backed Slot Antenna for IR-UWB in Air-Filled SIW Technology," in 2018 IEEE International Symposium on Antennas and Propagation USNC/URSI National Radio Science Meeting, pp. 12691270,2018 